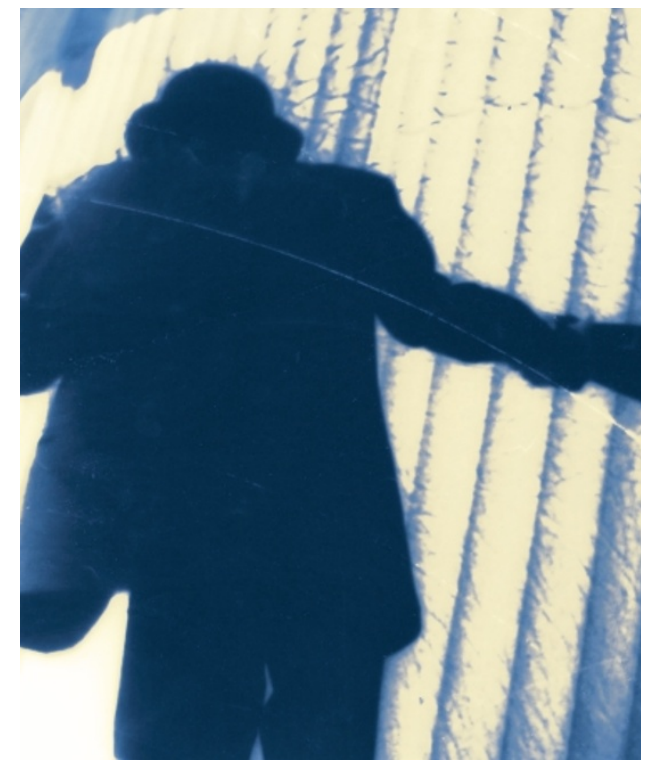

spread from monkeys to humans, how and when this occurred are questions of considerable debate.

HIV-2 closely resembles the strain of simian immunodeficiency virus (SIV) found in sooty mangabey monkeys. As explained in a report in the New Scientist. to investigate when this virus spread to humans, Dr. Ann-Mieke Vandamme and colleagues from the Katholieke Universiteit Leuven, Belgium, analysed the mutations present in HIV-2 and mangabey SIV and estimated the rate at which these would have accumulated. From this analysis, they concluded that HIV-2 probably crossed to humans as early as the 1940s.

AIDS caused by HIV-2 first occurred in Guinea-Bissau, and levels of infection remained low until the 1960s. As Vandamme told the Associated Press, the sharp increase in disease incidence coincided with the war to gain independence from Portugal between 1963 and 1974, and she speculated that the use of non-sterile injections and increased sexual activity during this time might have been important.

Not all of the coverage of this study has been positive, as reported by HealthScoutNews. Ernest Drucker, a professor of epidemiology at the Montefiore Medical Center and Albert Einstein College of Medicine in New York, has his doubts as to whether HIV-2 really spread to humans this early, and when asked about this work he said, 'The dates are very iffy'.

Jenny Buckland

\section{Partners in crime}

Bcll1a and Bcl11b, which encode zinc-finger transcription factors, are both associated with malignancies of the immune system. Gene-targeting studies, now published in Nature Immunology, indicate interesting differences between the roles of these paralogues in lymphocyte development.

Liu et al. first became interested in Bcl1 1a when they discovered that activation of this proto-oncogene by retroviral integration is associated with the development of myeloid leukaemia in mice. To investigate further the function of this gene, they generated $B c l 11 a^{-1-}$ mice, which died a few hours after birth. B cells were absent in the fetal livers of these mice, and fetal liver cells did not express genes that are normally expressed by B cells, including early

\section{IMMUNE EVASION}

\section{Germ warfare}

Imagine the scene. You are planning an invasion into enemy territory but know that the army waiting to defend against any attack is far bigger than your own small-scale operation. What you really need is a weapon that will wipe out a large number of the enemy in one go, without having to get involved in hand-to-hand combat. Staphylococcus aureus seems to have come up with an ideal solution. It secretes a protein that knocks out a large percentage of the B-cell repertoire in a supraclonal manner.

S. aureus protein $\mathrm{A}(\mathrm{SpA})$ is a B-cell superantigen that forms a complex with lymphocytes expressing B-cell receptors (BCRs) with clan-VHIIIencoded variable regions. These BCRs are displayed by $5-10 \%$ of mature mouse $B$ cells, including marginal-zone and follicular $B$ cells, and $B 1$ cells with a BCR antigenbinding region of the T15 idiotype. Now, Goodyear and Silverman have characterized the deletion of $B$ cells in response to this toxin in a study
B-cell factor 1 (Ebf1), paired box gene 5 (Pax5) and the interleukin-7 receptor (Il7r). Also, Bcl11 $a^{-/-}$fetal liver cells failed to develop into B cells after transplantation into lethally irradiated wild-type recipients. This indicates that Bcll1a is required for normal B-cell development and that it functions upstream of Ebf1 and Pax5.

In addition, T-cell development was disrupted in these mice, and when $B c l 11 a^{-1-}$ fetal liver cells were transferred into irradiated recipients, they failed to contribute normally to T-cell development. With age, most of the mice that had received $B c l 11 a^{-1-}$ cells developed leukaemia and died. Further experiments showed that the leukaemia cells were mainly of host origin, indicating that transplantation of $B c l 11 a^{-1-}$ cells resulted in tumorigenesis of host $\mathrm{T}$ cells, by some as-yet-unknown mechanism. This implies that normally, this tumoursuppressor gene functions in a noncell-autonomous manner - in other words, haematopoietic cells that express Bcl11a protect other cells from tumorigenesis. This paper confirms that, in addition to its roles in normal B- and T-cell development, Bcll1a can function as a tumour suppressor. published in The Journal of Experimental Medicine.

T15i immunoglobulin knock-in $\left(\mathrm{T} \mathrm{i}^{\mathrm{i} /+}\right)$ mice, in which almost all B cells express an $\mathrm{S107}$ (clan VHIII) $\mathrm{V}_{\mathrm{H}}$ transgene, were exposed to $\mathrm{SpA}$ and analysed after various time points. The initial response of $B$ cells to SpA was shown to be similar to the normal response to antigen exposure. After 2 hours, levels of the targeted cell-surface BCR were decreased; after 16 hours, the BCR co-receptors CD19 and $\mathrm{CD} 21$ were downregulated, and the activation markers CD69 and CD86 were upregulated; and after further in vitro culture, levels of MHC class II molecules and other markers were increased.

Next, the authors looked at whether the decrease in the number of $\mathrm{T}^{15 i^{+/+}} \mathrm{B}$ cells that follows this initial activation in response to SpA is owing to effects on trafficking or apoptosis. The adoptive transfer of labelled $\mathrm{T}^{15 \mathrm{i}^{+/+}}$splenocytes to $^{-}$ C57BL/6 recipients treated with SpA showed that after 48 hours of in vivo exposure, despite certain $B$ cells having undergone 2-3 rounds of proliferation, the number of T15i B cells was that splenic $\mathrm{T}^{15 \mathrm{i}^{+/+}} \mathrm{B}$ cells exposed to $\mathrm{SpA}$ undergo an increased rate of apoptosis, and this was also seen at other anatomical locations. After in vivo exposure to $\mathrm{SpA}$, levels of caspase- 3 were increased in parallel with the induction of DNA fragmentation. The inclusion of $\mathrm{Z}-\mathrm{VAD}$, a potent pan-caspase inhibitor, delayed the 'assisted suicide' of SpA-targeted T15i ${ }^{+/+}$ $\mathrm{B}$ cells. Bcl-2 overexpression, but not Fas (CD95) deficiency, was shown to rescue $B$ cells of SpA-treated $\mathrm{T}^{15 \mathrm{i}^{+/+}}$mice from apoptosis. The authors suggest that apoptosis in response to SpA is similar to the activation-induced cell death of $B$ cells in response to antigen. The authors are now hoping to put this microbial battle plan to therapeutic use. Up to $50 \%$ of human B cells express clan-VHIIIencoded BCRs and are therefore susceptible to SpA. Silverman and colleagues are engineering variants of SpA with higher binding affinities, and also with specificities for different framework variable regions, which will be evaluated for decreased by $\sim 36 \%$. This indicates 